\title{
Leptospirosis - Clinical and Echocardiographic Study
}

\author{
Sunisha Vinod L. ${ }^{1}$, Ajith S.N. ${ }^{2}$, Regi David S. ${ }^{3}$ \\ 1,2,3 Department of General Medicine, Government Medical College \\ (Affiliated under Kerala University of Health Sciences), Thiruvananthapuram, Kerala, India.
}

\section{ABSTRACT}

\section{BACKGROUND}

Leptospirosis is a worldwide zoonosis. It has a wide range of clinical symptoms ranging from asymptomatic subclinical infection to life-threatening multi-organ dysfunction. Among cardiac manifestations, myocarditis carries utmost importance as it is associated with poor outcomes in terms of morbidity and mortality. Definitive diagnosis of myocarditis by endomyocardial biopsy is difficult to popularize taking economic cost and operational complexity. We chose echocardiography as the imaging diagnosis method of myocarditis rather than cardiovascular magnetic resonance. Diagnosis of myocarditis arrived by using ECG, Echo and Cardiac enzymes. We wanted to estimate the proportion of cardiac involvement by clinical, electrocardiographical, serological and echocardiographical evaluation of patients with leptospirosis admitted in the Medicine department of Govt. Medical College, Thiruvananthapuram and the association of thrombocytopenia among patients with cardiac involvement.

\section{METHODS}

After obtaining institutional approval, 138 leptospirosis patients were enrolled for this study. Patients were interviewed and blood samples were taken for estimation of platelet count and cardiac enzymes. Electrocardiogram and echocardiogram were done, data entered in to excel sheet and analyzed using SPSS.

\section{RESULTS}

In this study out of 138 patients, $11.6 \%$ were having myocarditis as tested by using a combination of cardiac enzymes, ECG, Echo. Twelve percent of patients had echo findings suggestive of myocarditis. Mean platelet counts in patients with myocarditis were below 50000 .

\section{CONCLUSIONS}

Cardiac involvement is a serious manifestation occurring in leptospirosis. Besides cardiac MRI, echocardiogram serves as a useful imaging modality in the diagnosis of myocarditis. An electrocardiogram is the most sensitive investigation for the identification of myocarditis.

\section{KEY WORDS}

Leptospirosis, Myocarditis, Echo, ECG, Cardiac Enzymes, Cardiac Involvement.
Corresponding Author: Dr. Ajith S.N.,

H. No. B4, Chinmaya, TC-27/2363(1), Forest Office Lane B, Vazhuthacaud, Thiruvananthapuram-695014, Kerala, India.

E-mail: drajithsn@gmail.com

DOI: $10.14260 /$ jemds/2022/70

How to Cite This Article:

Vinod SL, AJith SN, David RS. Leptospirosis - clinical and echocardiographic study. J Evolution Med Dent Sci 2022;11(02):363368, DOI: 10.14260/jemds/2022/70

Submission 21-01-2022,

Peer Review 26-01-2022,

Acceptance 04-02-2022,

Published 07-02-2022.

Copyright (C) 2022 Sunisha Vinod L. et al. This is an open access article distributed under Creative Commons Attribution License [Attribution 4.0 International (CC BY 4.0)] 


\section{BACKGROUND}

Leptospirosis is an acute worldwide zoonosis caused by spirochaete Leptospira interrogans with clinical manifestations ranging from asymptomatic to severe shock and multi-organ dysfunction. Various factors involving rodents' environment and the occupational habits of humans can influence the transmission of the disease. ${ }^{1}$ It is an emerging public health problem especially for people who work habitually in wet occupations.

Fast diagnosis of leptospirosis and suitable antibiotic therapy along with supportive treatment are essential for the treatment of leptospirosis. Many leptospirosis cases are selflimited and the course of the disease is nonspecific, but it can result in a wide range of complications including renal involvement, icteric leptospirosis or Weil's syndrome, hypotension due to cardiovascular collapse.2,3 Changes in sensorium may also occur.

Medical literature records several studies demonstrating the extent of cardiac involvement in leptospirosis patients using clinical and echocardiographic methods. ${ }^{4,5}$ Several case studies are recording various electrocardiographic and echocardiographic findings in leptospirosis patients. $6,7,8$

Hence this study was planned for evaluating cardiac involvement in leptospirosis that would help understand pathophysiology and treatment modalities. The diagnosis of myocarditis is made based on the latest guidelines published by the European societies of cardiology.

Myocarditis being one of the serious manifestations of leptospirosis, early detection and treatment can improve the outcome.

\section{Objectives/Primary Objective}

To characterize the clinical profile, with particular emphasis on cardiovascular manifestations in patients with leptospirosis under the Department of Internal Medicine, Government Medical College, Thiruvananthapuram.

\section{Secondary Objectives}

1. To examine the correlation between thrombocytopenia and cardiac involvement

2. To ascertain the ECG manifestations and their correlation with echocardiographic abnormalities.

\section{METHODS}

It is a hospital-based cross-sectional study carried out at a tertiary care centre -Department of General Medicine, Government Medical College, Thiruvananthapuram from December 2018 to January 2021, after obtaining clearance from Institutional Human Ethics Committee. Informed consent was obtained from the participants.

\section{Study Population/Inclusion Criteria}

The study group consisted of patients with serologically diagnosed leptospirosis admitted in medicine wards of Govt medical college Trivandrum.

\section{Exclusion Criteria \\ 1. Prior cardiac disease. \\ 2. Prior similar ECG changes.}

\section{Budget}

No additional expenditure had been incurred from anyone for the study.

\section{Data Collection Tools}

Structured proforma including the relevant variables

\section{Data Collection Procedure}

After obtaining institutional ethics committee clearance, a minimum of 138 patients were enrolled on my study - all of them satisfied the inclusion criteria and gave consent to take part in the study. Relevant history of the case was sought and enquired specifically about symptoms of leptospirosis. All serologically diagnosed leptospirosis cases were taken. Cardiac symptoms were asked and the examination was done. Electrocardiograms and cardiac biomarkers of all diagnosed cases were assessed. The Trop t tests were done using Elecsys ${ }^{\circledR 2010}$ analyser (Roche Diagnostics, Mannheim, Germany). An echocardiogram of the patient was done. Thus cardiac functions were assessed. Among the patients with cardiac involvement, association of thrombocytopenia was then assessed.

\section{Data Analysis}

Data were entered into an excel sheet. Data analysis was done using appropriate statistical software. Categorical variables were expressed as proportions and quantitative variables as mean and standard deviation. Association was tested using the chi-square test. $\mathrm{P}<0.05$ was considered as statistically significant.

\section{RESULTS}

\section{Demographic Data}

\begin{tabular}{|cccccc|}
\hline & N & Minimum & Maximum & Mean & SD \\
Age in years & 138 & 22 & 89 & 46.65 & 12.80 \\
\hline \multicolumn{5}{c}{ Demographic Data } \\
\hline
\end{tabular}

66 patients (47.8\%) were males and $72(52.2 \%)$ were females.

Out of 138 patients, $56.5 \%$ were manual labourers, 35.5 $\%$ were semi-skilled and $8 \%$ were skilled.

Out of these patients, $30.4 \%$ were hypertensive and 42 (30.4\%) had dyslipidemia.

\section{Cardiac Symptoms \& Signs}

Out of 138 patients, $81.7 \%$ of them didn't have any cardiac symptoms and signs. The most common cardiac symptom was NYHA Class 2 and above dyspnoea 8 patients (5.8\%) followed by palpitations (4.3\%) and tachycardia (4.3\%). 3.6 $\%$ of patients had an ischemic type of chest pain. 


\begin{tabular}{|ccc|}
\hline ECG & Frequency & Percent \\
Normal ECG & 113 & 81.9 \\
Sinus tachycardia & 14 & 10.1 \\
Heart blocks & 4 & 2.9 \\
Dynamic ST T changes & 6 & 4.3 \\
Atrial fibrillation & 1 & 0.7 \\
Total & $\mathbf{1 3 8}$ & $\mathbf{1 0 0}$ \\
\hline Table 1. Distribution of the Sample According to Electrocardiography
\end{tabular}

Based on ECG findings, of the 138 patients, $18.1 \%$ had ECG changes, $10.1 \%$ had sinus tachycardia, $4.3 \%$ had dynamic ST -T changes, $2.9 \%$ had heart blocks, and $0.7 \%$ had atrial fibrillation.

\section{Positive Cardiac Biomarker (Troponin I)}

Trop I positivity was observed in sixteen (11.6\%) patients.

\begin{tabular}{|ccc|}
\hline Echocardiography & Frequency & Percent \\
Normal & 126 & 91.3 \\
Valvular regurgitant lesions & 2 & 1.4 \\
Wall motion abnormalities & 8 & 5.8 \\
LV systolic dysfunction & 2 & 1.4 \\
Total & $\mathbf{1 3 8}$ & $\mathbf{1 0 0}$ \\
\hline Table 2. Echocardiographic Abnormalities \\
\hline
\end{tabular}

Out of 138 patients, $8.6 \%$ had abnormal echo findings. $5.8 \%$ had wall motion abnormalities with global hypokinesia. $1.4 \%$ had valvular regurgitant lesions and $1.4 \%$ had LV systolic dysfunction.

\begin{tabular}{|ccc|}
\hline Platelet & Frequency & Percent \\
$>1.5$ & 1 & 0.7 \\
$1-1.5$ & 5 & 3.6 \\
$0.5-1.0$ & 98 & 71 \\
$0.2-0.5$ & 32 & 23.2 \\
$<0.2$ & 2 & 1.4 \\
Total & $\mathbf{1 3 8}$ & $\mathbf{1 0 0}$ \\
\hline Table 3. Distribution of the Sample According to Platelet Counts \\
\hline
\end{tabular}

The majority of the patients had platelet counts in between 50000 and 1 lakh. $1.4 \%$ had platelet counts < 20000.

In (10.14 \%) patients with cardiac symptoms, echocardiogram was normal. 11 symptomatic patients had echocardiographic evidence of cardiac involvement.

Out of patients having wall motion abnormalities, $60 \%$ were in the age group of 30 to $60 \mathrm{yrs}$.

\begin{tabular}{|c|c|c|c|c|c|c|c|c|c|c|}
\hline \multirow{3}{*}{ ECG } & \multicolumn{8}{|c|}{ Echocardiography } & \multirow{2}{*}{\multicolumn{2}{|c|}{ Total }} \\
\hline & \multicolumn{2}{|c|}{ 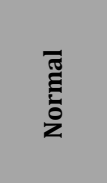 } & \multicolumn{2}{|c|}{ 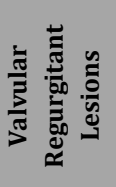 } & \multicolumn{2}{|c|}{ 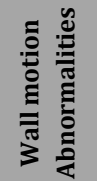 } & \multicolumn{2}{|c|}{ 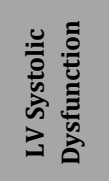 } & & \\
\hline & $\mathbf{n}$ & $\%$ & $\mathbf{n}$ & $\%$ & $\mathbf{n}$ & $\%$ & $\mathbf{n}$ & $\%$ & $\mathbf{n}$ & $\%$ \\
\hline Normal ECG & 112 & 88.9 & 1 & 50 & 0 & 0 & 0 & 0 & 113 & 81.9 \\
\hline Sinus tachycardia & 9 & 7.1 & 1 & 50 & 3 & 37.5 & 1 & 50 & 14 & 10.1 \\
\hline Heart blocks & 2 & 1.6 & 0 & 0 & 2 & 25 & 0 & 0 & 4 & 2.9 \\
\hline $\begin{array}{l}\text { Dynamic ST T } \\
\text { changes }\end{array}$ & 3 & 2.4 & 0 & 0 & 2 & 25 & 1 & 50 & 6 & 4.3 \\
\hline Atrial fibrillation & 0 & 0 & 0 & 0 & 1 & 12.5 & 0 & 0 & 1 & 0.7 \\
\hline Total & 126 & 100 & 2 & 100 & 8 & 100 & 2 & 100 & 138 & 100 \\
\hline
\end{tabular}

In a study population of 138, $112(81.1 \%)$ patients had normal ECG and echo findings. 1 of the patients had echo suggestive of myocarditis with normal ECG. In 14 (10.14\%) patients with ECG suspicious of myocarditis had normal Echo. Whereas 11 (7.9\%) patients had ECG and Echo suggestive of myocarditis.
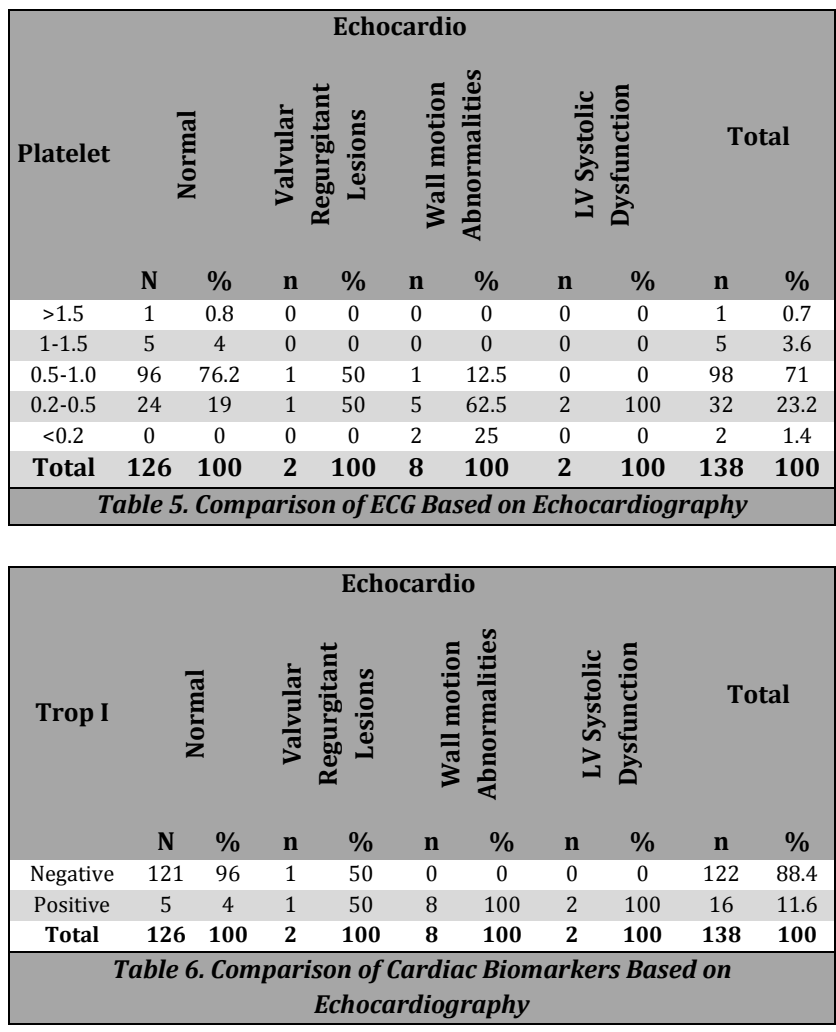

\begin{tabular}{|c|c|c|c|c|c|c|c|c|c|c|}
\hline \multirow{3}{*}{$\begin{array}{c}\text { Clinical } \\
\text { Cardiology }\end{array}$} & \multicolumn{8}{|c|}{ Echocardiography } & \multirow{2}{*}{\multicolumn{2}{|c|}{ Total }} \\
\hline & \multicolumn{2}{|c|}{ हृ } & \multicolumn{2}{|c|}{ 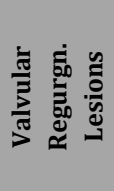 } & \multicolumn{2}{|c|}{ 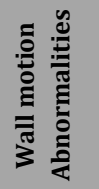 } & \multicolumn{2}{|c|}{ 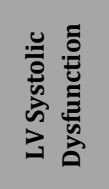 } & & \\
\hline & $\mathbf{n}$ & $\%$ & n & $\%$ & $\mathbf{n}$ & $\%$ & n & $\%$ & $\mathbf{n}$ & $\%$ \\
\hline Normal & 112 & 88.9 & 1 & 50 & 0 & 0 & 0 & 0 & 113 & 81.9 \\
\hline $\begin{array}{l}\text { Ischemic type } \\
\text { chest pain }\end{array}$ & 3 & 2.4 & 0 & 0 & 2 & 25 & 0 & 0 & 5 & 3.6 \\
\hline Palpitations & 4 & 3.2 & 0 & 0 & 2 & 25 & 0 & 0 & 6 & 4.3 \\
\hline $\begin{array}{l}\text { NYHA class } 2 \text { and } \\
\text { above dyspnoea }\end{array}$ & 5 & 4 & 0 & 0 & 3 & 37.5 & 0 & 0 & 8 & 5.8 \\
\hline Tachycardia & 2 & 1.6 & 1 & 50 & 1 & 12.5 & 2 & 100 & 6 & 4.3 \\
\hline Total & 126 & 100 & 2 & 100 & 8 & 100 & 2 & 100 & 138 & 100 \\
\hline
\end{tabular}

Out of 138 patients, 121 (87.68\%) patients with negative cardiac biomarkers had a normal echo. 5 patients with positive biomarkers also had a normal echo. 1 patient with a normal cardiac biomarker had echo finding suggestive of the acute regurgitant lesion. 11(7.9\%) patients had elevated cardiac biomarkers and echocardiographic changes.

Patients with low platelet count had more associated myocarditis as evidenced from echo. One could see that every patient with myocarditis had a platelet count of $<50000$ and $100 \%$ of patients with platelet count $<20000$ had abnormal echocardiogram as evidenced by wall motion abnormalities

\section{DISCUSSION}

Leptospirosis is caused by pathogenic spirochetes of the genus leptospira. It was Weil, Professor of Medicine at Heidelberg (1886) whose name has been given to the disease in humans. He first reported this disease which is caused by Leptospira interrogans, serovar icterohaemorrhagiae or 
copenhageni. ${ }^{9}$ Its infectious nature and microbial origin were proved independently, first in Japan by Inada et al. (Spirochaeta icterohaemorrhagiae) in 1915.10

Leptospirosis is a worldwide zoonosis. According to the different nature of the disease presentations, different names have been used, e.g. seven-day fever found commonly in Japan, Cane cutter's disease in Australia, Rice field Leptospirosis in Indonesia and Fort Bragg fever in the US. Weil's disease, which is the severe form of this disease, occurs in many countries, including South-East Asian Countries, India, China, continental Europe and England. Leptospirosis exists in all five inhabited continents. It occurs in tropical, subtropical, and temperate zones. ${ }^{2}$

\section{Cardiac Involvement in Leptospirosis}

The clinical spectrum of leptospirosis is wide with a mild febrile illness to severe multi-organ dysfunction state. Cardiac manifestations are well documented in leptospirosis [4] and they range from non-specific electrocardiographic changes and arrhythmias to myocarditis, pericarditis, endocarditis and cardiogenic shock. ${ }^{11}$

\section{Pathophysiology}

The pathophysiology of cardiac involvement in leptospirosis is very little studied. Tissue damage in leptospirosis is an outcome of several interactions between the bacteria and host defence mechanisms. Mechanism posted was direct damage to tissues by leptospires resulting in a sepsis-like syndrome with immune activation, and a systemic vasculitis to result in widespread damage. 12

There is definite evidence of cardiac pathology in patients dying of severe leptospirosis.5,13 Histopathological evidence of damage and inflammation in the heart was demonstrated in these post mortem studies. Interstitial myocarditis with cellular infiltrate was observed in leptospirosis patients (in respective studies as $96 \%, 593.2 \%, 450 \% 14$

Perivascular inflammation affecting the intramural and the major coronary arteries was demonstrated in the autopsy. Even though echocardiographic findings were different, in autopsies, cardiomegaly was also a noted feature. Right heart dilatation was postulated to be secondary to pulmonary haemorrhage and respiratory failure. This proves histopathological evidence of cardiac inflammation and vasculitis. This may be due to systemic inflammatory response or specific response to leptospira. Systemic vasculitis is a possible mechanism for organ dysfunction in leptospirosis. ${ }^{12}$ In this context, the presence of cardiac inflammation and vasculitis is highly significant.

\section{Diagnostic Criteria of Myocardial Involvement} There are no clear criteria for diagnosing cardiac involvement in leptospirosis. Cardiac symptoms like chest pain, dyspnoea, pulmonary oedema, arrhythmias, refractory shock.15-18 Hypotension and shock may be due to factors other than direct cardiac involvement and may even be the major cause, like septic shock, severe acidosis from acute renal involvement, haemorrhage ${ }^{6}$ and autonomic involvement. ${ }^{19}$ But the refractory shock is considered to indicate myocardial involvement. ${ }^{15,16}$

\section{Electrocardiographic Abnormalities in Leptospirosis}

Although electrocardiographic abnormalities can be easily studied in every leptospirosis patient, they have low specificity for cardiac involvement in leptospirosis. Electrolyte abnormalities and deranged homeostasis may result in ECG changes without direct cardiac involvement by the disease. ${ }^{7}$ A wide variety of ECG changes are seen and ECG changes are thought to occur very early in the course. ECG changes include nearly the full spectrum of abnormalities, including sinus tachycardia and relative and marked bradycardias, ${ }^{219}$ bundle branch blocks, P-QRS-T complex changes, low voltage QRS, ${ }^{8}$ ST-T changes, intraventricular conduction blocks, non-specific ventricular repolarization changes, ${ }^{8,18}$ ventricular and supraventricular extrasystoles, first-degree and third-degree AV blocks and atrial fibrillations. ${ }^{18,19}$

\section{Echocardiographic Evidence of Myocardial Dysfunction}

Myocardial functional changes are most easily assessed by echocardiography nowadays. In echo cardiac assessment, two studies demonstrated normal left ventricular function.

\section{Cardiac Biomarkers Study: Evidence of Myocardial Damage}

Creatine kinase myocardial band levels were shown to be elevated in patients with leptospirosis in one study. But, the correlation between the finding of CK-MB levels and clinical cardiac involvement was poor, and CK-MB levels could not be used as a reliable biomarker for cardiac involvement. Elevated troponin levels have been demonstrated in a cohort study, but their significance is not known. Concluding, the place of cardiac biomarkers in identifying or predicting cardiac involvement in leptospirosis is unknown.

\section{Endocarditis}

It has been reported as an extremely rare complication of leptospirosis. ${ }^{20}$ Wang et al. reported a patient with leptospirosis who developed cardiac aneurysm, brain abscess and liver and kidney infarcts. Leptospira spirochaetes were demonstrated in biopsy specimens of this patient's excised cardiac tissue (aneurysm and mitral valve), as well as in needle biopsy specimens of renal, liver and brain tissue. But in this case, blood cultures also showed methicillin-resistant Staphylococcus aureus. Whether the manifestations were the result of co-infection with this organism could not be found out.

The observations were made on 138 leptospirosis patients admitted to the medical wards.

The study had 66 males and 72 females constituting a male to female ratio of $0.91: 1$. The mean age was found to be $46.65 \pm 12.80$. About 42 patients had hypertension and dyslipidemia. Out of 138 patients, $81.7 \%$ patients did not have any cardiac symptoms or signs. The most common cardiac symptom was NYHA Class 2 and above dyspnoea (5.8 $\%$ ) followed by palpitations (4.3\%) and tachycardia. $3.6 \%$ of patients had the ischemic type of chest pain. 
In a study conducted by Tharanga Fernando et al. (2013) ten (45\%) patients complained of cardiac symptoms (ischemic type chest pain [10, $100 \%]$, palpitations [6, $60 \%]$ and new-onset shortness of breath corresponding to NYHA classification II and above [2, 20 \%]). Tachycardia (heart rate. $100 / \mathrm{min})$ was documented on admission in $6(27 \%)$ patients (pulse rate. 100). Rajiv C et al. (1996) studied cardiovascular involvement in 50 patients with serologically proved leptospirosis. Twelve (24\%) patients had dyspnoea and 18 (36\%) had transient hypotension during the illness.

Except for 1 patient, all of them had a platelet count $<1.5$ lakhs. The majority of the patients had platelet counts in between 50000 and 1 lakh. $1.4 \%$ had platelet counts < 20000. Based on ECG findings, of the 138 patients, $18.1 \%$ had ECG changes, $10.1 \%$ had sinus tachycardia, $4.3 \%$ had dynamic ST -T changes, $2.9 \%$ had heart blocks, and $0.7 \%$ had atrial fibrillation.

In our study out of 138 patients, $11.6 \%$ had raised troponin I > $0.02 \mathrm{ng} / \mathrm{ML}$ while Anoop Mathew et al. (2018) demonstrated $61 \%$ of critically ill leptospirosis patients in their ICUs to be trop T positive. In a study by Jayathilaka et al. (2019), all 5 patients showed elevated cardiac biomarkers.

Out of 138 patients, $12 \%$ had abnormal echocardiographic findings. $5.8 \%$ had wall motion abnormalities with global hypokinesia. $1.4 \%$ had valvular regurgitant lesions, $1.4 \%$ had LV systolic dysfunction. Echocardiographic abnormalities were seen in $41 \% .{ }^{9}$ Clinical and echocardiographic evidence of myocarditis was seen in 2 patients but left ventricular function was preserved into Tharanga Fernando et al. (2013). They concluded that echocardiographic changes may be useful in identifying patients with myocarditis in leptospirosis, especially in symptomatic patients. Rajiv C et al. (1996) ${ }^{6}$ Left ventricular function as assessed by echocardiography and Doppler examination was normal. Anoop Mathew et al. (2018) demonstrated that myocardial systolic dysfunction was common in both groups (leptospirosis: $55.86 \%$ vs. sepsis syndrome: $51.61 \%$ ) with subclinical left ventricular systolic dysfunction characterized by abnormal global longitudinal strain and normal left ventricular ejection fraction being most frequent followed by isolated right ventricular systolic dysfunction, isolated left ventricular systolic dysfunction, and bi-ventricular systolic dysfunction (leptospirosis: $31.43 \%$, $18.42 \%, 13.16 \%, 10.53 \%$, respectively.

In this study of 138 patients, 112 (81.1\%) had normal ECG and echo findings. 1 of the patients had echo suggestive of myocarditis with normal ECG. 14 (10.14\%) patients with ECG suspicious of myocarditis had normal echo. Whereas 11 (7.9\%) patients had ECG and echo suggestive of myocarditis. Out of 138 patients, 121 (87.68\%) patients with negative cardiac biomarkers had a normal echo. 5 patients with positive biomarkers also had a normal echo. 1 patient with a normal cardiac biomarker had echo finding suggestive of the acute regurgitant lesion. 11 (7.9\%) patients had elevated cardiac biomarkers and echocardiography.

The mean platelet count of leptospirosis patients with normal echo was 67230.2. Among patients with echocardiographic abnormalities, those with valvular regurgitant lesions were 44500 . While that of wall motion abnormalities were 31500. Patients with LV systolic dysfunction had a platelet count of 35500 . Echocardiographic evidence of cardiac involvement was seen in patients with low platelet counts.

In this study, we found out that of 138 patients, 25 patients had electrocardiographic evidence, 16 had positive cardiac biomarker elevation and 12 patients had echocardiographic evidence of cardiac involvement.

\section{CONCLUSIONS}

The diagnosis of myocarditis was arrived at as per European society of cardiology guidelines ${ }^{21}$ by using study tools like ECG, cardiac enzymes, echocardiography.

The following conclusions were made from this study.

- Cardiac signs and symptoms were present in $18.3 \%$ of patients, but these were nonspecific.

- $\quad$ The proportion of myocarditis was $18.1 \%$ as evidenced by ECG changes, $11.59 \%$ by elevated cardiac enzymes and $\quad 8.69 \%$ by echocardiography. Thus electrocardiogram was the most sensitive test in the detection of myocarditis. Sinus tachycardia was the most common electrocardiographic abnormality.

- Regarding echocardiographic findings, Wall motion abnormalities with global hypokinesia were the most common. There was a strong association between myocarditis and severe thrombocytopenia.

- This study demonstrated the clinical and diagnostic characteristics of leptospirosis associated myocarditis and its association with platelet counts. Clinical manifestations of leptospiral myocarditis primarily were arrhythmias and heart failure, which would often lead to some serious consequences, such as shock and death.

\section{REFERENCES}

[1] World Health Organization. Leptospirosis burden epidemiology reference group (LERG). https://www.who.int/zoonoses/diseases/lerg/en/

[2] Sehgal SC. Epidemiological patterns of leptospirosis. Indian J Med Microbiol 2006;24(4):310-1.

[3] Centers for Disease Control and Prevention [CDC]. Leptospirosis [online]

[4] Chakurkar G, Vaideeswar P, Pandit SP, et al. Cardiovascular lesions in leptospirosis: an autopsy study. J Infect 2008;56(3):197-203.

[5] Shah K, Amonkar GP, Kamat RN, et al. Cardiac findings in leptospirosis. J Clin Pathol 2010;63(2):119-23.

[6] Rajiv C, Manjuran RJ, Sudhayakumar N, et al. Cardiovascular involvement in leptospirosis. Indian Heart J 1996;48(6):691-4.

[7] Ramachandran S. Electrocardiographic abnormalities in leptospirosis. J Trop Med Hyg 1975;78:210-3.

[8] Skerk V, Markotic A, Puljiz I, et al. Electrocardiographic changes in hospitalized patients with leptospirosis over a 10-year period. Med Sci Monit 2011;17(7):CR369-75.

[9] Howie J. Virology, and Immunity. In: Topley and Wilson's principles of bacteriology. Vol. 38. $8^{\text {th }}$ edn. London: Edward Arnold 1985. 
[10] Inada R, Ido Y, Hoki R, et al. The etiology, mode of infection, and specific therapy of well's disease (spirochaetosis icterohaemorrhagica). J Exp Med 1916;23(3):377-402.

[11] Navinan MR, Rajapakse S. Cardiac involvement in leptospirosis. Transactions of the Royal Society of Tropical Medicine and Hygiene 2012;106(9):515-20.

[12] Cinco M. New insights into the pathogenicity of leptospires: evasion of host defences. New Microbiol 2010;33(4):283-92.

[13] Segura ER, Ganoza CA, Campos K, et al. Clinical spectrum of pulmonary involvement in leptospirosis in a region of endemicity, with quantification of leptospiral burden. Clin Infect Dis 2005;40(3):343-51.

[14] de Brito T, Morais CF, Yasuda PH, et al. Cardiovascular involvement in human and experimental leptospirosis: pathologic findings and immunohistochemical detection of leptospiral antigen. Ann Trop Med Parasitol 1987;81(3):207-14.

[15] Boignard A, Bonadona A, Hamidfar R, et al. Cardiogenic shock due to acute myocarditis complicating leptospirosis. Arch Mal Coeur Vaiss 2006;99(3):251-4.
[16] Singh SS, Vijayachari P, Sinha A, et al. Clinicoepidemiological study of hospitalized cases of severe leptospirosis. Indian J Med Res 1999;109:94-9.

[17] Guivarch G, Le Gall JR, Regnier B JF. Shock during leptospirosis icterohaemorrhagica. Nouv Presse Med 1982;11:837-9.

[18] Dussarat GV, Cointet F, Capdevielle P, et al. Cardiac manifestations in leptospirosis. Apropos of 15 cases observed in New Caledonia. Ann Cardiol Angeiol (Paris) 1988;37:449-53.

[19] Chen HI, Kao SJ, Hsu YH. Pathophysiological mechanism of lung injury in patients with leptospirosis. Pathology 2007;39(3):339-44.

[20] Caignault JR, Staat P, Poncet B, et al. Icterohaemorrhagic leptospirosis with a cardiac presentation in a patient returning from an endemic zone [article in French]. Arch Mal Coeur Vaiss 2006;(99):259-61.

[21] Caforio ALP, Pankuweit S, Arbustini E, et al. Current state of knowledge on aetiology, diagnosis, management and therapy of myocarditis: a position statement of the European Society of Cardiology Working Group on Myocardial and Pericardial Diseases. Eur Heart J 2013;34(33):2636-2648, 48a-48d. 